\title{
The Creative Task Creator: A tool for the generation of customized, Web-based creativity tasks
}

\author{
Jean E. Pretz And John A. Link \\ Illinois Wesleyan University, Bloomington, Illinois
}

\begin{abstract}
This article presents a Web-based tool for the creation of divergent-thinking and open-ended creativity tasks. A Java program generates HTML forms with PHP scripting that run an Alternate Uses Task and/or open-ended response items. Researchers may specify their own instructions, objects, and time limits, or use default settings. Participants can also be prompted to select their best responses to the Alternate Uses Task (Silvia et al., 2008). Minimal programming knowledge is required. The program runs on any server, and responses are recorded in a standard MySQL database. Responses can be scored using the consensual assessment technique (Amabile, 1996) or Torrance's (1998) traditional scoring method. Adoption of this Web-based tool should facilitate creativity research across cultures and access to eminent creators. The Creative Task Creator may be downloaded from the Psychonomic Society's Archive of Norms, Stimuli, and Data, www.psychonomic.org/archive.
\end{abstract}

In this article, we describe the Creative Task Creator, a Java-based tool that generates software that can be used for Web-based administration of creativity tasks, including the Alternate Uses Task (Guilford, 1950), Silvia's modification of this task (Silvia et al., 2008), and an open-ended free-response task. The Creative Task Creator is creative in two senses: It not only generates tasks, but also allows for flexibility in the design of those tasks. Use of the Creative Task Creator will facilitate the collection of data from various populations, including participants across cultures and expert creators. Use of the software will also streamline the scoring process, since all responses are collected in a digital format in a common database.

\section{MEASURING CREATIVITY}

Researchers have been attempting to measure creativity and creative potential since the mid-20th century. Guilford (1950) admonished the APA to undertake the task of studying creativity seriously, which required the development of materials for measuring the construct. On the basis of his structure of intellect model of intelligence, Guilford (1967) advocated for several measures of divergent thinking ability. Expanding on the work of Guilford, Torrance (1998) developed a battery of such tasks, the Torrance Tests of Creative Thinking, which are still widely used in the field. One of these tests, the Alternate Uses Task, simply asks participants to list as many uses as possible for a common everyday object, such as a brick. Torrance also included figural tasks such as the Circles Task, in which participants make drawings out of circles that are printed on a page.
Divergent-thinking tasks are known for their ease of administration and objectivity in scoring. Such tasks require little background knowledge and can be administered to participants of any age or background. They can also be administered in groups, which facilitates the datacollection process. Responses can be scored according to objective criteria such as fluency (number of ideas generated), originality (uniqueness of ideas), and flexibility (variability in categories of ideas).

More recently, creativity researchers have turned to measuring creativity by using open-ended tasks that are rated for creativity by judges. Rather than using contentgeneral divergent-thinking tasks with questionable predictive validity for later creative achievement, researchers have begun using tasks that prompt participants to create complete works, which are then rated for their creativity. Tasks have included collage making, essay writing, and even caption writing for cartoons. These tasks are scored using Amabile's (1996) consensual assessment technique (CAT). This scoring system asks independent raters to judge each work according to their own subjective definitions of creativity. Amabile has argued that high rates of interrater reliability indicate that there is a consensus among judges with respect to their definitions of creativity. This process has become very popular among creativity researchers. When relying on the CAT method, participants can create actual artworks, researchers can measure creativity in a specific domain, and judges can evaluate creativity using a process that is more ecologically valid than the traditional objective scoring methods used by Torrance (1998).

J. E.Pretz, jpretz@iwu.edu 
A new idea in creativity measurement is a hybrid model proposed by Silvia et al. (2008), who modified the Alternate Uses Task to take into account each participant's own definition of what is highly creative. In this method, participants complete a traditional Alternate Uses Task and then indicate the top two ideas from their own list. These ideas are then rated for creativity, using the CAT. Silvia has argued that this method identifies participants who not only can generate many novel ideas, but also can identify which of their ideas are truly the most creative. Runco (1991) has also argued that the ability to evaluate ideas is critical for real-life creative pursuits.

No matter which method of creativity measurement one adopts, the assessment of creativity is often onerous and cumbersome. When traditional paper-and-pencil administration is used, responses to divergent-thinking tasks must be processed prior to scoring. Given that participants can generate dozens of responses for each prompt, the process of data management can become extremely time consuming. Typically, the responses must be transcribed into a digital format, which requires researchers to decipher the handwriting and correct the spelling in thousands of responses. By their very nature, these steps increase the possibility that data will be compromised when researchers are forced to make judgments about the intended responses of participants. Creativity measures using the CAT involve similar problems. Because researchers using the CAT typically desire a more ecologically valid creative product, they must allow participants a fair amount of time to produce each creative work. These works must then be processed in preparation for the rating process. The Creative Task Creator software will facilitate the processing of data in these creativity tasks. Responses to divergentthinking and CAT tasks are typed directly by participants and are stored in a digital format that can be downloaded for scoring at the researcher's convenience.

\section{Creativity Tasks on the Web}

The Creative Task Creator allows researchers with minimal programming knowledge to generate their own customized software for Web-based creativity tasks. As with any Web-based research, researchers who use this software can collect data from anyone with access to the Internet, enabling them to recruit participants who can be difficult to bring into the lab. Using the Creative Task Creator, researchers can create multiple Alternate Uses items, tailor their own instructions, specify a time limit, and request that participants select their best responses from those that they have generated. In addition, researchers can create their own open-ended, free-response items. Such items can prompt participants to create an essay based on a given title, image, or sound. Examples used previously in the literature include Sternberg and Lubart's (1995) instructions to create an essay with the title "2983" and Kaufman's (2002) use of photographs to inspire creative responses. The possibilities for using this free-response prompt are limited only by the creativity of the researcher.
A major benefit of this software is that the responses from all participants are stored in a single, consolidated, digital database. Web-based administration ensures that data collected at multiple sites are stored in the same database. As mentioned above, storing responses electronically facilitates the export of data to a spreadsheet format for scoring or rating, eliminating the need for a lengthy and error-prone transcription process.

\section{The Creative Task Creator}

The Creative Task Creator is an open-source, Java-based program that runs on any platform that supports Java (Version 1.5 or later). Researchers must have a host server with server-side PHP and MySQL functionality. Server space may be available through universities or departments, or the researcher may choose to pay a minimal fee for a professional Web-hosting service such as netfirms.com. Basics on how to conduct Web-based research, including details on how to set up a server through Netfirms, have been described by Fraley (2007). Information on the best practices in conducting Internet research can be found in an article by Reips (2002).

To use the Creative Task Creator, researchers can download the files from www.iwu.edu/ jpretz/creativity.html (or from the Psychonomic archive) and specify their menu options using the CreativeTaskCreator.jar program. The program generates the tasks in the form of HTML files that implement PHP scripting. The Creative Task Creator provides point-and-click programming capability for researchers with no programming background. Experienced programmers can insert HTML code into any of the application's text inputs, or they can edit the created files directly. (See Figure 1 for an image of the Java interface.)

The Creative Task Creator allows researchers to design an Alternate Uses Task according to their own specifications. As a default, the program includes three Alternate Uses objects (brick, chair, and deck of cards). Researchers can use these default objects, specify up to nine of their own, or eliminate the Alternate Uses Task altogether. The default settings allow participants to provide up to 50 alternate uses for each object, although this number can be edited by the researcher. Researchers can modify the default instructions to suit their own purposes, including instructional manipulations. Researchers may also set a time limit for participant responses in the Alternate Uses Task. The default time limit is set at $3 \mathrm{~min}$, but researchers can specify limits of up to 100 min or have no time limit at all.

In addition, researchers can ask participants to designate which of their responses to the Alternate Uses Task they consider their best responses. Silvia et al. (2008) asked participants to identify the top two responses, but researchers can modify this number as well. To allow participants to designate their best responses, the program automatically reproduces the list of participant-generated Alternate Use responses, with a checkbox next to each. Participants then mark the checkboxes that correspond to their selections. The specific instructions for selecting top responses can also be edited. The program automati- 


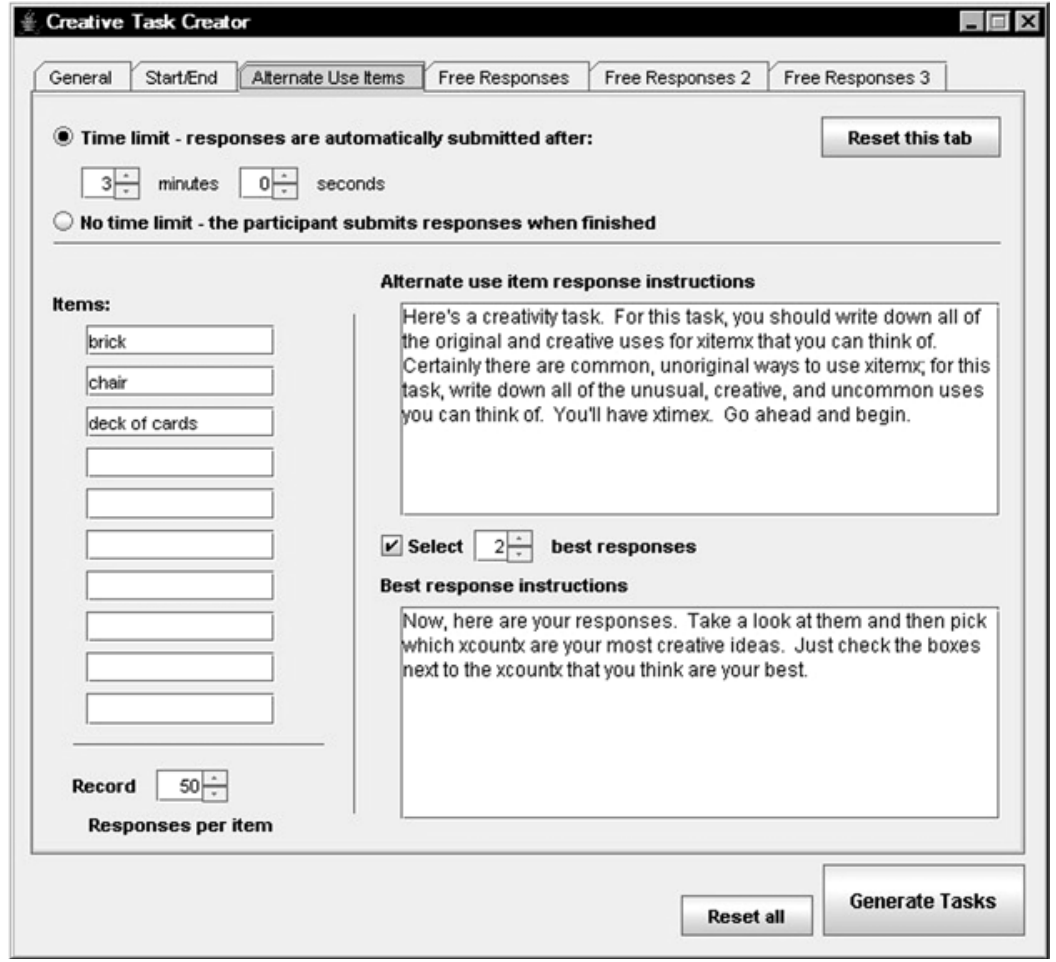

Figure 1. Creative Task Creator interface.

cally adjusts the task instructions to reflect the researcherspecified number of objects, time limit, and number of top responses.

The Creative Task Creator also facilitates the design of an open-ended task with researcher-specified instructions. The default instructions for this task reflect a domainspecific creativity task called the Dream Project Task (Landers \& Pretz, 2008). Participants are prompted to describe a dream project in their major field of study, given unlimited time and resources. This task was created to complement the domain-general divergent-thinking tasks by soliciting creativity in the domain of training. The default time limit for this free-response task is $10 \mathrm{~min}$. Researchers can choose to include up to 11 free-response items, with the possibility of different instructions for each. The time limit can also be eliminated; participants are asked instead to submit their responses when they are done. This very versatile prompt can be used to initiate any creativity task with written responses or simply to ask for comments on the Alternate Uses Task.

After the researcher has designed the tasks, the Creative Task Creator generates the customized software files and stores them in a folder with the default name "creativitytask." These files contain the task specifications and key information about the MySQL server settings (host, database, data table, user, and password). To initiate the creativity tasks, researchers must open the file called "taskstart.php." The functionality of the tasks, including time limits, can be tested offline; however, no data will be recorded until the files are linked to the MySQL database on the server.
To implement the tasks online, researchers must upload the entire task folder to their server and integrate the task with their introductory Web page by creating a link to the taskstart.php file. This link initiates the customized creativity software, and the MySQL data table is automatically created when the first participant completes the tasks.

Results are stored in the MySQL database that is hosted on the researcher's server in an automatically generated table that is specific to the tasks. Researchers may then output the data as they wish for scoring and/or rating. The MySQL code is easily identifiable within each PHP script, so that a skilled programmer can make minor modifications to record data in a Microsoft SQL or PostgreSQL database, or in another data format.

Data for each participant can be identified using a unique participant ID number. As a default, the MySQL database automatically records a unique "counter" variable for each participant in the table. Researchers preferring to specify ID numbers for their data collection can enter them at the beginning of each testing session (if testing in a laboratory and in person) or have participants generate a unique identifier on the spot. If participants are off-site and need to enter a specific ID number, researchers can provide instructions on the introductory page that explain how to create a unique participant ID. The ID field can also be left blank. A warning to researchers, however: Repeat ID numbers are not detected by the program.

The Creative Task Creator is designed for creating tasks and recording data, not for scoring responses. Researchers who are interested in scoring the responses online should 
consult Ames et al. (2005) for a suitable and flexible Webbased program. The use of Web-based ratings allows for the entire process, from data collection through scoring, to be conducted off-site. Participants and raters can be recruited to contribute from anywhere in the world.

\section{DISCUSSION}

This article presents open-source software for the Webbased implementation of widely used creativity tasks, including divergent-thinking tasks and open-ended freeresponse tasks. To our knowledge, no other Web-based tool for creativity research has been available previously. Furthermore, the Creative Task Creator affords researchers a great deal of flexibility in tailoring the tasks to their own research needs without requiring expertise in programming.

This software eases the process of lab-based data collection. Participants can be brought into the lab and asked to complete the materials online. The resultant data are stored in a digital format and in a single database, ready to be downloaded. When researchers bring participants into the lab, they can provide prompts that are not easily presented on the computer screen (e.g., a physical prop as the subject for brainstorming alternate uses). Researchers can also include additional creativity tasks in the protocol (e.g., collage making).

We hope that the Creative Task Creator can provide a starting point for researchers who want to go beyond the basic administration of these creativity tasks. One limitation of the software is that it is currently designed to solicit only written responses from participants. Savvy researchers may find the structure of this software to be a valuable template for creating additional tasks, such as the figural divergent-thinking tasks used by Torrance (1998). An additional limitation is that Creative Task Creator does not allow for the specification of experimental conditions. Researchers who want to introduce a manipulation into their study (e.g., instructional manipulations) should be able to do so with a bit of creativity and programming know-how. Specifically, researchers can create unique versions of the creativity tasks to correspond to the experimental conditions in the study and can administer them to participants in a randomized or fixed order.

Our hope is that the Creative Task Creator will facilitate longitudinal research on creativity, research across cultures, and research with expert creators. Using this tool, researchers may find it easier to recruit the same individuals at multiple time points across development. A primary critique of divergent-thinking measures of creativity has been their apparent lack of predictive validity (Plucker \& Renzulli, 1999); therefore, longitudinal work is critical for establishing the predictive validity of these creativity tasks.

The use of Web-based creativity tasks might help to bridge geographical gaps that stand in the way of increased understanding of creativity across cultures. Recent work has found that the effects of cultural factors on creativity do not parallel their effects on reasoning tasks (Niu, Zhang, \& Yang, 2007). Using the Creative Task Creator, researchers can streamline the recruitment of participants from multiple international sites while maintaining a consistent research protocol and a single database of responses.

Finally, another major challenge in the field has been the need to gain access to eminent creators. Most research with highly creative individuals has relied primarily on case-study methods (Csikszentmihalyi, 1996) and historiometric analyses (Simonton, 1999a). Simonton (1999b) advocated the expanded use of "significant samples," samples that include eminent individuals, such as those with great creative success. Web-based data collection may enable researchers to entice these creators to complete some standardized measures for comparison with the responses of less eminent creators on their campuses. Once researchers are able to compare eminent and typical individuals on the same task, more experimental work with eminent samples should be possible.

This tool for the creation of Web-based creativity tasks should facilitate research in the field by expanding access to diverse samples, simplifying the processing of data, and facilitating the scoring process. The Creative Task Creator can be used to generate an infinite number of creativity tasks with written responses. We encourage researchers to take advantage of this versatility and to be creative in designing new tasks.

\section{AUTHOR NOTE}

This work was supported by an Illinois Wesleyan University Junior Faculty Leave to J.E.P. Many thanks to Paul Silvia for sharing with us his new protocol for assessing creativity using divergent-thinking tasks, and for encouraging us to publish this Web-based implementation of it. We also thank Craig Landers for his feedback in developing the Dream Project task. J.A.L. is a 2007 graduate of the Illinois Wesleyan Math and Computer Science department. Correspondence concerning this article should be addressed to J. E. Pretz, Psychology Department, Illinois Wesleyan University, P.O. Box 2900, Bloomington, IL 61701 (e-mail: jpretz@iwu.edu).

\section{REFERENCES}

Amabile, T. M. (1996). Creativity in context. Boulder, CO: Westview Press.

Ames, S. L., Gallaher, P. E., Sun, P., Pearce, S., Zogg, J. B., Houska, B. R., ET AL. (2005). A Web-based program for coding open-ended response protocols. Behavior Research Methods, 37, 470-479.

Csikszentmihalyi, M. (1996). Creativity: Flow and the psychology of discovery and invention. New York: HarperCollins.

Fraley, R. C. (2007). Using the Internet for personality research: What can be done, how to do it, and some concerns. In R. W. Robins, R. C. Fraley, \& R. F. Krueger (Eds.), Handbook of research methods in personality psychology (pp. 130-148). New York: Guilford.

GuILford, J. P. (1950). Creativity. American Psychologist, 5, 444-454.

Guilford, J. P. (1967). The nature of human intelligence. New York: McGraw-Hill.

Kaufman, J. C. (2002). Narrative and paradigmatic thinking styles in creative writing and journalism students. Journal of Creative Behavior, 36, 201-220.

LAnders, C., \& Pretz, J. E. (2008). Development of domain-general and domain-specific creativity among college students. Unpublished manuscript. 
NiU, W., Zhang, J. X., \& YAng, Y. (2007). Deductive reasoning and creativity: A cross-cultural study. Psychological Reports, 100, 509-519.

Plucker, J. A., \& Renzulli, J. S. (1999). Psychometric approaches to the study of human creativity. In R. J. Sternberg (Ed.), Handbook of creativity (pp. 35-61). New York: Cambridge University Press.

ReIPS, U.-D. (2002). Standards for Internet-based experimenting. Experimental Psychology, 49, 243-256.

Runco, M. A. (1991). The evaluative, valuative, and divergent thinking of children. Journal of Creative Behavior, 25, 311-319.

Silvia, P. J., Winterstein, B. P., Willse, J. T., Barona, C. M., Cram, J. T., Hess, K. I., ET AL. (2008). Assessing creativity with divergent thinking tasks: Exploring the reliability and validity of new subjective scoring methods. Psychology of Aesthetics, Creativity, \& the Arts, 2, 68-85.

Simonton, D. K. (1999a). Creativity from a historiometric perspective. In R. J. Sternberg (Ed.), Handbook of creativity (pp. 116-133). New York: Cambridge University Press.

Simonton, D. K. (1999b). Significant samples: The psychological study of eminent individuals. Psychological Methods, 4, 425-451.

Sternberg, R. J., \& Lubart, T. I. (1995). Defying the crowd: Cultivating creativity in a culture of conformity. New York: Free Press.

Torrance, E. P. (1998). Torrance Tests of Creative Thinking: Normstechnical manual: Figural (streamlined) forms $A$ and B. Bensenville, IL: Scholastic Testing Service.

\section{ARCHIVED MATERIALS}

The following materials may be accessed through the Psychonomic Society's Norms, Stimuli, and Data archive, www.psychonomic.org/ archive.

To access these files, search the archive for this article using the journal name (Behavior Research Methods), the first author's name (Pretz), and the publication year (2008).

FILE: Pretz-BRM-2008.zip

DESCRIPTION: The compressed archive file contains five files and folders:

CreativeTaskCreator.jar, the executable file.

The img folder, containing fr1.jpg, item1.jpg, item1checks.jpg, tasken .jpg, and taskstart.jpg.

The lib folder, containing swing-layout-1.0.3.jar.

The src folder, containing CreativeTaskCreator.form and Creative TaskCreator.java.

readme.html, providing information about Creative Task Creator.

AUTHOR's E-MAIL ADDRESs: jpretz@iwu.edu

(Manuscript received February 27, 2008; revision accepted for publication April 2, 2008.) 